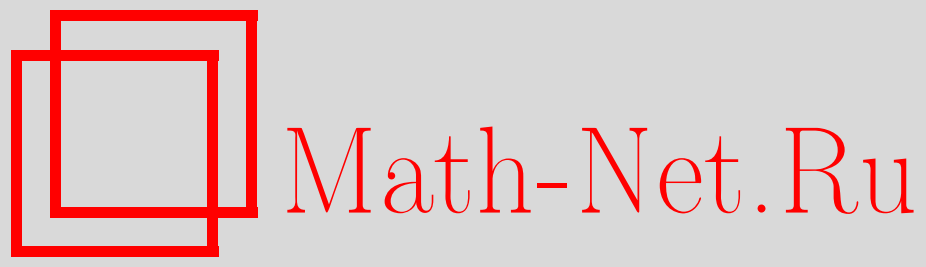

Ю. М. Березанский, А. А. Мохонько, Интегрирование некоторых дифференциально-разностных нелинейных уравнений с помощью спектральной теории блочных якобиевых нормальных матриц, Функи. анализ и его прил., 2008, том 42, выпуск 1, 1-21

DOI: https://doi.org/10.4213/faa2886

Использование Общероссийского математического портала MathNet.Ru подразумевает, что вы прочитали и согласны с пользовательским соглашением

http://www . mathnet.ru/rus/agreement

Параметры загрузки:

IP : 35.173 .137 .237

26 апреля 2023 г., 14:33:34

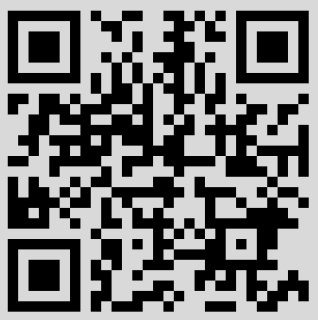




\title{
Интегрирование некоторых дифференциально-разностных нелинейных уравнений с помощью спектральной теории блочных якобиевых нормальных матриц*
}

\author{
(c) 2008. Ю. М. БЕРезАнСКй̆, А. А. Мохонько \\ Светлой памяти М. Г. Крейна в связи со 100-летием его рождения
}

Для интегрирования задачи Коши по времени $t$ для нелинейного уравнения Кортевега-де-Фриза имеется известный метод обратной спектральной задачи для самосопряженного уравнения Штурма-Лиувилля на полуоси, предложенный и развитый в классических работах И. М. Гельфанда, Б. М. Левитана, В. А. Марченко и М. Г. Крейна (см. его изложение в [1]). Пользуясь некоторыми результатами для конечной цепочки Тоды (см. [2], [3]), один из авторов в [4][6] предложил аналогичный метод интегрирования задачи Коши по $t$ для разностного нелинейного уравнения - полубесконечной цепочки Тоды, основывающийся на более простой спектральной теории разностного аналога уравнения Штурма-Лиувилля - полубесконечной самосопряженной якобиевой матрицы. Подчеркнем, что в обоих случаях использовались самосопряженные операторы.

Главная цель этой работы - показать, что аналогичная теория интегрирования соответствующих классов нелинейных разностных уравнений может быть развита на основании недавно построенной в [7], [8] спектральной теории нормальных полубесконечных якобиевых матриц. При этом подходе с необходимостью появляются матричные (неабелевы) нелинейные разностные уравнения. Это связано не со стремлением к обобщениям, а с тем жестким фактом, что нормальные трехдиагональные матрицы - это обязательно матрицы с блочной якобиевой структурой с (как правило) нарастающими конечными размерностями блоков.

Теперь несколько слов о спектральной теории блочных самосопряжсенных якобиевых матриц. Эта теория была создана под влиянием заметки М. Г. Крейна 1949 г. [9], развивалась, в частности, в работах [10]-[12] и нашла применение в интегрировании задачи Коши для некоторых классов матричных разностных уравнений [13]-[16] (применялись также некоторые результаты типа спектральных относительно общих блочных якобиевых матриц, развитые в [17], [18]). Ситуация здесь оказалась менее приятной: решение основополагающего дифференциального уравнения по $t$ для аналога функции Вейля можно было найти лишь в достаточно специальных случаях.

Остановимся еще на одном аспекте рассматриваемой задачи, когда разностная переменная меняется на всей оси $\mathbb{Z}=\{n\}=\{\ldots,-1,0,1, \ldots\}$, а не на полуоси. В этом случае даже решение задачи Коши для обычной цепочки Тоды вызывает серьезные трудности: если применить метод удвоения [12] и свести

\footnotetext{
*Работа выполнена при частичной поддержке грантом DFG 436 UKR 113/78/0-1.
} 
рассмотрение к разностному уравнению на полуоси, но для блочной $(2 \times 2)$ якобиевой матрицы, то проинтегрировать уравнение по $t$ относительно функции Вейля не удается. Здесь имеется большое число работ (как в скалярном, так и в неабелевом матричном случае), в которых в специальных ситуациях и при специальных исходных данных эти трудности удается преодолеть, используя не только спектральный подход. Так, в периодическом случае уравнение было проинтегрировано в терминах тэта-функций в [19] (см. также [20]), методом обратной задачи теории рассеяния (для разностной задачи) - в [21]-[23], подобным используемому в [4]-[6] методом для убывающих к нулю при $|n| \rightarrow \infty$ начальных данных - в [24]-[26]; см. также [27]-[29]. Имеется также большое количество работ в указанных направлениях, носящих более физический характер. С некоторыми из них можно познакомиться в сборнике трудов конференции, фигурирующей в [30]. Отметим также недавно появившуюся книгу [31], содержащую математически корректное изложение ряда вопросов интегрирования задачи Коши для нелинейных разностных уравнений.

Остановимся несколько более детально на методе работ [4]-[6] интегрирования задачи Коши для цепочки Тоды на полуоси при помощи спектральной теории классических якобиевых матриц. Его суть сводилась к тому, что с решением $u(t), t \in[0, \infty)$, связывалась определенным простым образом ограниченная в пространстве $\ell_{2}$ самосопряженная якобиева матрица $J(t)$, эволюция спектральной меры $d \rho(\lambda ; t)$ которой во времени легко подсчитывалась. Поэтому по спектральной мере $d \rho(\lambda ; 0)$ матрицы $J(0)$, отвечающей начальному значению $u(0)$ решения, легко было подсчитать $d \rho(\lambda ; t)$ для произвольного $t>0$, а значит, и восстановить при помощи решения обратной спектральной задачи саму матрицу $J(t)$ и тем самым найти решение $u(t)$.

В дальнейшем в статьях [32], [33], [30], [34] этот метод был обобщен на неизоспектральные уравнения, для которых спектр матрицы $J(t)$ изменяется во времени (для цепочки Тоды он от $t$ не зависит). В [35] был рассмотрен случай, когда якобиева матрица $J(t)$ порождает неограниченный (но самосопряженный) оператор в $\ell_{2}$.

В последние годы усилился интерес к теории ортогональных полиномов на единичной окружности (см. [36], [37]). Такие полиномы связаны с операторами, действующими в обычном пространстве $\ell_{2}$ и порождающимися пятидиагональными унитарными матрицами (вместо трехдиагональных самосопряженных якобиевых) [38]. Оказалось, что и в этом случае спектральную теорию таких матриц можно применить, подобно самосопряженному случаю, к интегрированию некоторых нелинейных уравнений [39]. Роль цепочки Тоды теперь играет поток Шура.

Как уже говорилось, недавно была развита спектральная теория блочных нормальных якобиевых матриц [7], [8]. Они действуют в пространстве $\mathbf{l}_{2}$, которое равно ортогональной сумме конечномерных (а не одномерных, как в случае $\ell_{2}$ ) пространств, и порождают нормальный оператор.

В частности, этот оператор может быть унитарным. Тогда он действует в пространстве $\mathbf{l}_{2, u}=\mathbb{C}^{1} \oplus \mathbb{C}^{2} \oplus \mathbb{C}^{2} \oplus \cdots \subset \mathbf{l}_{2}$ и задается блочной якобиевой матрицей. Если воспринимать пространство $\mathbf{l}_{2, u}$ как обычное пространство $\ell_{2}$ (после введения стандартного базиса в $\mathbb{C}^{2}$ ), то этот оператор задается пятидиагональной матрицей, которая совпадает с рассматривавшейся в [36]-[39]. Развитая в [7], [8] теория аналогична классической теории якобиевых матриц: обобщенные 
собственные векторы будут последовательностью полиномов от $z$ и $\bar{z}$, ортогональных относительно спектральной меры на плоскости (или, для унитарного случая, на единичной окружности).

Повторим, что целью этой работы является перенесение подхода работ [4]-[6] на случай блочных якобиевых матриц, порождающих нормальный (или унитарный) оператор в пространстве $\mathbf{l}_{2}$ (или $\mathbf{l}_{2, u}$ ). В унитарном случае результаты близки к полученным в [39].

В 11 излагается без доказательств спектральная теория блочных якобиевых нормальных матриц [8]. Для ее лучшего понимания в начале параграфа мы кратко напоминаем ситуацию с классическими якобиевыми матрицами. $\S \S 2,3$ посвящены интегрированию нелинейных разностных уравнений в случае, когда спектр исходного нормального оператора одномерен, а $\$ 5$ - когда он двумерен. В $\$ 4$ мы кратко излагаем ситуацию, когда в роли нормальной блочной якобиевой матрицы выступает ее частный случай - унитарная блочная якобиева матрица, применяя при этом соответствующую спектральную теорию [7], с ортогональными полиномами на единичной окружности. Подобный подход в терминах пятидиагональных унитарных матриц в обычном пространстве $\ell_{2}$ был предложен Голинским в [39] как развитие подхода из [4]-[6]. Статья [39] стимулировала написание предлагаемой работы.

\section{§1. Спектральная теория блочных якобиевых нормальных матриц}

1. Классическая спектральная теория самосопряженных якобиевых матриц строится в пространстве $\ell_{2}$ последовательностей $f=\left(f_{n}\right)_{n=0}^{\infty}, f_{n} \in \mathbb{C}$. Оператор задается трехдиагональной (якобиевой) матрицей $J$ с элементами $\left(b_{n}\right)_{n=0}^{\infty}$ на главной диагонали и $\left(a_{n}\right)_{n=0}^{\infty}$ по двум смежным, $b_{n} \in \mathbb{R}, a_{n}>0$. Он обозначается через $\boldsymbol{J}$ и строится как замыкание в $\ell_{2}$ отображения $\ell_{2} \supset \ell_{\text {fin }} \ni f \mapsto J f \in \ell_{2}$. Этот оператор будет эрмитовым и, при определенном поведении на $\infty$ элементов $a_{n}, b_{n}$, самосопряженным (если $a_{n}, b_{n}$ равномерно ограничены, то $\boldsymbol{J}-$ ограниченный самосопряженный оператор).

Разложение по обобщенным собственным векторам $\left(P_{n}(\lambda)\right)_{n=0}^{\infty}$ оператора $\boldsymbol{J}$ $\left(P_{n}(\lambda) \in \mathbb{R}, \lambda \in \mathbb{R}\right)$ строится следующим образом. Рассмотрим разностное уравнение

$$
(J f)_{n}=a_{n-1} f_{n-1}+b_{n} f_{n}+a_{n} f_{n+1}=\lambda f_{n}, \quad n \in \mathbb{N}_{0}, f_{-1}=0,
$$

на полуоси $\mathbb{N}_{0}=\{0\} \cup \mathbb{N}$, где $\lambda \in \mathbb{R}-$ спектральный параметр. Благодаря условию $a_{n}>0$ всегда существует решение $f_{n}=P_{n}(\lambda)$ уравнения (1) (являющееся полиномом степени $n$ от $\lambda)$. Последовательность $P(\lambda)=\left(P_{n}(\lambda)\right)_{n=0}^{\infty}$ будет в известном смысле обобщенным собственным вектором оператора $\boldsymbol{J}$, отвечающим собственному значению $\lambda, \boldsymbol{J} P(\lambda)=\lambda P(\lambda)$.

Соответствующее преобразование Фурье ${ }^{\wedge}$ имеет вид

$$
\ell_{2} \supset \ell_{\text {fin }} \ni f=\left(f_{n}\right)_{n=0}^{\infty} \mapsto \widehat{f}(\lambda)=\sum_{n=0}^{\infty} f_{n} P_{n}(\lambda) \in L^{2}(\mathbb{R}, d \rho(\lambda))=: L^{2} .
$$

После замыкания по непрерывности отображение (2) становится унитарным оператором, переводящим пространство $\ell_{2}$ в пространство $L^{2}$ суммируемых с квадратом функций относительно спектральной меры $d \rho(\lambda)$ оператора $\boldsymbol{J}$ (борелевской вероятностной меры с бесконечным числом точек роста). При преобразовании Фурье (2) оператор $\boldsymbol{J}$ переходит в оператор умножения на $\lambda$ в $L^{2}$. 
Эта конструкция тесно связана с теорией ортогональных полиномов от $\lambda$ на вещественной оси относительно спектральной меры. А именно, из (2) следует, что $\left(P_{n}(\lambda)\right)_{n=0}^{\infty}$ образуют ортонормированный полиномиальный базис в $L^{2}$. Считая спектральную меру $d \rho(\lambda)$ заданной, этот базис можно построить непосредственно: нужно применить классическую процедуру ортогонализации Шмидта в пространстве $L^{2}$ к последовательности линейно независимых функций

$$
1, \lambda, \lambda^{2}, \ldots, \lambda^{n}, \ldots, \quad \lambda \in \mathbb{R}
$$

(считается, что система (3) линейно независима и полна в $L^{2}$ ). В результате ортогонализации мы получим последовательность $P(\lambda)=\left(P_{n}(\lambda)\right)_{n=0}^{\infty}$ - обобщенную собственную функцию оператора $\boldsymbol{J}$, спектральной мерой которого является заданная мера $d \rho(\lambda)$. Для элементов $a_{n}, b_{n}$ матрицы $J$ теперь выписываются простые формулы. В этом заключается простейшая обратная задача спектрального анализа: каким образом по заданной спектральной мере $d \rho(\lambda)$ восстановить оператор $\boldsymbol{J}$, спектральной мерой которого будет заданная мера $d \rho(\lambda)$.

2. Спектральная теория блочных якобиевых нормальных матриц (см. [7], [8]) строится аналогично описанной выше классической теории. Сейчас вместо пространства $\ell_{2}$ рассматривается гильбертово пространство

$$
\begin{array}{cc}
\mathbf{l}_{2}=\mathscr{H}_{0} \oplus \mathscr{H}_{1} \oplus \cdots, & \mathscr{H}_{n}=\mathbb{C}^{n+1}, n \in \mathbb{N}_{0}, \\
\mathbf{l}_{2} \ni f=\left(f_{n}\right)_{n=0}^{\infty}, \quad & \sum_{n=0}^{\infty}\left\|f_{n}\right\|_{\mathscr{H}_{n}}^{2}<\infty .
\end{array}
$$

Векторы $x_{n}$ из пространства $\mathscr{H}_{n}=\mathbb{C}^{n+1}$ удобно обозначать так: $x_{n}=\left(x_{n ; 0}, \ldots\right.$, $\left.x_{n ; n}\right)$. Для любого $\alpha \in\{0, \ldots, n\}$ число $x_{n ; \alpha} \in \mathbb{C}$ является координатой при базисном векторе

$$
\delta_{n ; \alpha}=(0, \ldots, 0,1,0, \ldots, 0) \in \mathscr{H}_{n} \subset \mathbf{l}_{2}
$$

(единица стоит на $\alpha$-м месте), $\delta_{0}=(1)$. Обобщение якобиевой матрицы сейчас выглядит следующим образом $\left(n \in \mathbb{N}_{0}\right)$ :

$$
J=\left(\begin{array}{cccccc}
b_{0} & c_{0} & 0 & 0 & 0 & \ldots \\
a_{0} & b_{1} & c_{1} & 0 & 0 & \ldots \\
0 & a_{1} & b_{2} & c_{2} & 0 & \ldots \\
\vdots & \vdots & \vdots & \vdots & \vdots & \ddots
\end{array}\right), \quad \begin{aligned}
& a_{n}: \mathscr{H}_{n} \rightarrow \mathscr{H}_{n+1}, \\
& \mathscr{H}_{n}: \mathscr{H}_{n+1} \rightarrow \mathscr{H}_{n} \\
& \mathscr{H}_{n}
\end{aligned}
$$

Такая матрица (5) на финитных векторах из $\mathbf{l}_{\text {fin }} \subset \mathbf{l}_{2}$ индуцирует оператор

$$
\mathbf{l}_{2} \supset \mathbf{l}_{\text {fin }} \ni f \mapsto J f=\left((J f)_{n}\right)_{n=0}^{\infty} \subset \mathbf{l}_{2}, \quad(J f)_{n}=a_{n-1} f_{n-1}+b_{n} f_{n}+c_{n} f_{n+1} .
$$

Здесь $f_{-1}=0$. Предполагается, что этот оператор допускает замыкание, которое будет обозначаться через $\boldsymbol{J}$.

Как легко видеть, формально сопряженная к $J$ матрица $J^{+}$имеет ту же структуру (5), но с элементами $c_{n}^{+}, b_{n}^{+}$и $a_{n}^{+}$на нижней, средней и верхней диагоналях; матрица $J^{+}$однозначно определяется по $J$ соотношением $(J f, g)_{\mathbf{l}_{2}}=$ $\left(f, J^{+} g\right)_{\mathbf{l}_{2}}, f, g \in \mathbf{l}_{\text {fin }}$ (для числовых матриц ${ }^{+}$означает обычное сопряжение).

Для простоты мы будем предполагать, что нормы всех матриц $a_{n}, b_{n}, c_{n}$ равномерно ограничены; тогда оператор (6) ограничен. Таким же будет аналогично построенный (по $\left.J^{+}\right)$оператор $\boldsymbol{J}^{+}$. 
Основное ограничение в настоящей статье: матрица $J$ формально нормальная: $J J^{+}=J^{+} J$ (нетрудно выписать соотношения на элементы матриц $a_{n}, b_{n}$, $c_{n}$, обеспечивающие такую нормальность). В случае равномерно ограниченных элементов матрицы $J$ оператор $\boldsymbol{J}$ будет нормальным: $\boldsymbol{J}^{*} \boldsymbol{J}=\boldsymbol{J} \boldsymbol{J}^{*}$.

Роль положительности $a_{n}$ в обычной теории якобиевых матриц сейчас будут играть более громоздкие условия, обеспечивающие разрешимость уравнений типа (1) для нашего случая. А именно, будем предполагать, что выполнено следующее условие: околодиагональные матрицы имеют вид

$$
\begin{aligned}
& a_{n}=\left(\begin{array}{cccccc}
a_{n ; 0,0} & a_{n ; 0,1} & a_{n ; 0,2} & \cdots & a_{n ; 0, n-1} & a_{n ; 0, n} \\
0 & a_{n ; 1,1} & a_{n ; 1,2} & \cdots & a_{n ; 1, n-1} & a_{n ; 1, n} \\
0 & 0 & a_{n ; 2,2} & \cdots & a_{n ; 2, n-1} & a_{n ; 2, n} \\
\vdots & \vdots & \vdots & \ddots & \vdots & \vdots \\
0 & 0 & 0 & \cdots & a_{n ; n-1, n-1} & a_{n ; n-1, n} \\
0 & 0 & 0 & \cdots & 0 & a_{n ; n, n} \\
0 & 0 & 0 & \cdots & 0 & 0
\end{array}\right) \underbrace{}_{n+1}
\end{aligned}
$$

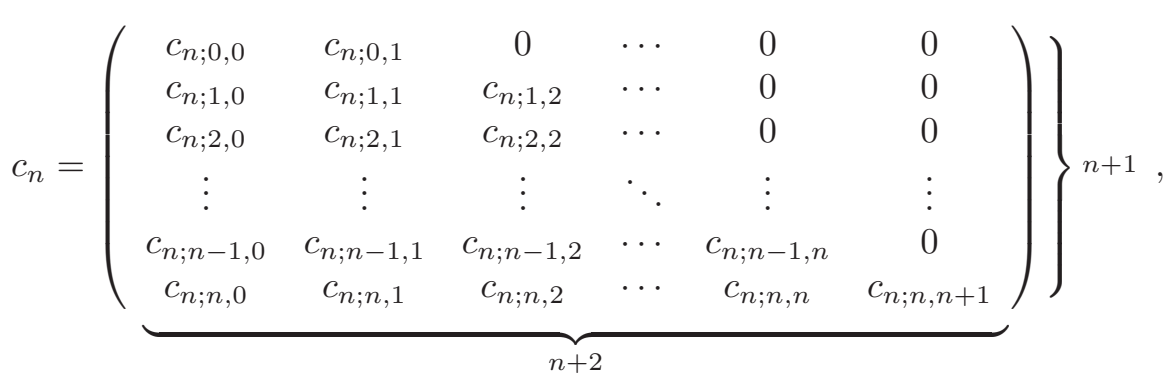

$$
\begin{aligned}
& a_{n ; 0,0}, a_{n ; 1,1}, \ldots, a_{n ; n, n} ; c_{n ; 0,1}, c_{n ; 1,2}, \ldots, c_{n ; n, n+1}>0, \quad n \in \mathbb{N}_{0} .
\end{aligned}
$$

Роль обобщенного собственного вектора $P(\lambda)=\left(P_{n}(\lambda)\right)_{n=0}^{\infty}$ сейчас играет последовательность $P(z)=\left(P_{n}(z)\right)_{n=0}^{\infty}$, где $P_{n}(z) \in \mathscr{H}_{n}$ - векторнозначный полином от $z, \bar{z}$ порядка $n, z \in \mathbb{C}, n \in \mathbb{N}_{0}$. А именно

$$
P_{n}(z)=\left(\overline{P_{n ; 0}(z)}, \overline{P_{n ; 1}(z)}, \ldots, \overline{P_{n ; n}(z)}\right),
$$

где $P_{n ; \alpha}(z), \alpha=0, \ldots, n,-$ некоторая линейная комбинация с комплексными коэффициентами функций

$$
1 ; z^{1} \bar{z}^{0}, z^{0} \bar{z}^{1} ; \ldots ; z^{n} \bar{z}^{0}, z^{n-1} \bar{z}^{1}, \ldots, z^{n-\alpha} \bar{z}^{\alpha}
$$

(коэффициент при $z^{n-\alpha} \bar{z}^{\alpha}$ положителен).

Полиномы (8) являются решениями двух разностных уравнений:

$$
J P(z)=z P(z), \quad J^{+} P(z)=\bar{z} P(z) \quad \forall z \in \mathbb{C} .
$$

Отметим, что, в отличие от (1), здесь появляются два уравнения, так как для нормального оператора $z, \bar{z}$ одновременно будут собственными значениями. Условия (7) являются, подобно условию $a_{n}>0$, условиями разрешимости уравнений (9).

Как и в классическом случае, для построения $P_{n}(z)$ можно использовать процедуру классической ортогонализации Шмидта в пространстве $L^{2}:=L^{2}(\mathbb{C}, d \rho(z))$ 
относительно заданной борелевской вероятностной меры $d \rho(z)$, ортогонализуя вместо простой последовательности (3) более сложную:

$$
z^{0} \bar{z}^{0} ; z^{1} \bar{z}^{0}, z^{0} \bar{z}^{1} ; z^{2} \bar{z}^{0}, z^{1} \bar{z}^{1}, z^{0} \bar{z}^{2} ; \ldots ; z^{n} \bar{z}^{0}, z^{n-1} \bar{z}^{1}, \ldots, z^{0} \bar{z}^{n} ; \ldots, \quad z \in \mathbb{C} .
$$

Kaк и ранее, предполагается, что система (10) линейно независима и полна в $L^{2}$ (например, это будет так, когда носитель меры $d \rho(z)$ является замыканием ограниченного открытого множества из $\mathbb{C}$ ).

Результат ортогонализации удобно записать в виде следующей схемы, согласованной с последовательностью (10):

$$
\begin{aligned}
& P_{0 ; 0}(z) \equiv 1 ; \quad P_{1 ; 0}(z), \quad P_{2 ; 0}(z), \quad \cdots \quad P_{n ; 0}(z), \quad \cdots \\
& P_{1 ; 1}(z) ; \quad P_{2 ; 1}(z), \quad \cdots \quad P_{n ; 1}(z), \ldots \\
& P_{2 ; 2}(z) ; \quad \cdots \quad P_{n ; 2}(z), \quad \cdots \\
& \text { … } \cdots \\
& P_{n ; n}(z) ; \quad \cdots
\end{aligned}
$$

Тогда по определению каждый столбец в (11), взятый с комплексной чертой, образует вектор $P_{n}(z)$ согласно (8).

Роль произведения $f_{n} P_{n}(\lambda)$ в преобразовании Фурье (2) сейчас будет играть скалярное произведение:

$\left(f_{n}, P_{n}(z)\right)_{\mathscr{H}_{n}}=f_{n ; 0} \overline{P_{n ; 0}(z)}+f_{n ; 1} \overline{P_{n ; 1}(z)}+\cdots+f_{n ; n} \overline{P_{n ; n}(z)}, \quad f_{n} \in \mathscr{H}_{n}, n \in \mathbb{N}_{0}$,

где $f_{n ; \alpha}$ - координаты вектора $f_{n}$ в соответствии с (4).

3. Спектральная теорема для нормального (ограниченного) оператора $\boldsymbol{J}$, порождаемого в пространстве $\mathbf{l}_{2}$ матрицей $(5)$, сейчас будет выглядеть следующим образом [8].

Теорема 1. Пусть $J$ - бесконечная блочная нормальная якобиева матрииа (5), нормы всех элементов которой равномерно ограничены и для которой выполнено условие (7). Она порождает в пространстве $\mathbf{1}_{2}$ ограниченный нормальный оператор как замыкание по непрерывности отображения (6).

Преобразование Фуръе по обобщенным собственным функииям оператора $\boldsymbol{J}$ uмеет вид

$$
\begin{aligned}
\mathbf{l}_{2} \supset \mathbf{l}_{\text {fin }} \ni f=\left(f_{n}\right)_{n=0}^{\infty} \\
\quad \mapsto \widehat{f}(z)=\sum_{n=0}^{\infty}\left(f_{n}, P_{n}(z)\right)_{\mathscr{H}_{n}}=\sum_{n=0}^{\infty} \sum_{\alpha=0}^{n} f_{n ; \alpha} \overline{P_{n ; \alpha}(z)} \in L^{2}(\mathbb{C}, d \rho(z))=: L^{2},
\end{aligned}
$$

где $d \rho(z)$ - спектральная мера оператора $\boldsymbol{J}$, являющаяся борелевской вероятностной мерой на $\mathbb{C}$ с компактным носителем. В формуле $(13) P(z)=$ $\left(P_{n}(z)\right)_{n=0}^{\infty}$ является обобщенным собственным вектором оператора $\boldsymbol{J}$, отвечающим $z \in \mathbb{C}$. Здесь $P_{n}(z) \in \mathscr{H}_{n}$ - векторнозначные полиномы от $z$ u $\bar{z}$ указанного в (8) вида.

После замыкания по непрерывности отображсение (13) становится унитарным оператором `между пространствами $\mathbf{l}_{2}$ u $L^{2}$. Образом оператора $\boldsymbol{J}\left(\boldsymbol{J}^{*}\right)$ при этом отображсении будет оператор умножсения на $z(\bar{z})$ в пространстве $L^{2}$. Равенство Парсеваля имеет вид

$$
(f, g)_{\mathbf{l}_{2}}=\int_{\mathbb{C}} \widehat{f}(z) \overline{\widehat{g}(z)} d \rho(z), \quad(\boldsymbol{J} f, g)_{\mathbf{l}_{2}}=\int_{\mathbb{C}} z \widehat{f}(z) \overline{\widehat{g}(z)} d \rho(z) \quad \forall f, g \in \mathbf{l}_{2} .
$$


Полиномь $\overline{P_{n ; \alpha}(z)}, n \in \mathbb{N}, \alpha=0, \ldots, n, u P_{0 ; 0}(z) \equiv 1$ образуют в пространстве $L^{2}$ полную ортонормированную систему в следующем смысле:

$$
\begin{array}{r}
\int_{\mathbb{C}}\left(\overline{P_{j ; 0}(z)} f_{j ; 0}+\cdots+\overline{P_{j ; j}(z)} f_{j ; j}\right)\left(P_{k ; 0}(z) \overline{g_{k ; 0}}+\cdots+P_{k ; k}(z) \overline{g_{k ; k}}\right) d \rho(z) \\
=\delta_{j, k}\left(f_{j}, g_{k}\right)_{\mathscr{H}_{j}} \quad \forall f_{j} \in \mathscr{H}_{j}, g_{k} \in \mathscr{H}_{k}, j, k \in \mathbb{N}_{0} .
\end{array}
$$

Матрица $J=\left(a_{j, k}\right)_{j, k=0}^{\infty}, a_{j, k}=\left(a_{j, k ; \alpha, \beta}\right)_{\alpha, \beta=0}^{j, k}$, восстанавливается по формуле

$$
a_{j, k ; \alpha, \beta}=\left(J \delta_{k ; \beta}, \delta_{j ; \alpha}\right)_{\mathbf{l}_{2}}=\int_{\mathbb{C}} z \overline{P_{k ; \beta}(z)} P_{j ; \alpha}(z) d \rho(z) .
$$

Таким образом, прямая спектральная задача для оператора $\boldsymbol{J}$ состоит в нахождении его спектральной меры $d \rho(z)$ и обобщенных собственных векторов $P(z)=\left(P_{n}(z)\right)_{n=0}^{\infty}, z \in \mathbb{C}$, которые ищутся как решения уравнений (9) указанного в (8) вида.

Обратная спектральная задача состоит в нахождении оператора $\boldsymbol{J}$, т. е. матрицы (5), удовлетворяющей условиям (7), по заданной спектральной мере $d \rho(z)$ в $\mathbb{C}$. Для ограниченного нормального оператора $\boldsymbol{J}$ она формулируется следующим образом [8]:

Теорема 2. Пусть $d \rho(z)$ - заданная борелевская вероятностная мера на $\mathbb{C}$ с компактным носителем, для которой система функиий (10) линейно независима и полна в пространстве $L^{2}(\mathbb{C}, d \rho(z)$ ) (например, пусть носитель этой меры содержнт открытую в $\mathbb{C}$ область или расположен на одномерном многообразии из $\mathbb{C}$ и не сводится $\kappa$ конечному множеству точек). Тогда мера $d \rho(z)$ обязательно является спектральной для некоторого ограниченного нормального оператора $\boldsymbol{J}$, порожсденного блочной якобиевой нормальной матрицей $J$ вида (5), удовлетворяющей условиям (7).

Элементы этой матрицы восстанавливаются по формуле (16), где полиномы $P_{n ; \alpha}(z), \alpha=0, \ldots, n, n \in \mathbb{N}_{0}$, строятся по системе (10) описанной выше классической процедурой ортогонализачии по Шмидту.

Если исходная мера $d \rho(z)$ была спектральной для некоторого оператора $\boldsymbol{J}$, порожденного блочной якобиевой нормальной матрищей $J$ с равномерно ограниченными нормами элементов, то восстановленная таким образом по $d \rho(z)$ матрица будет совпадать с J.

\section{§2. Уравнение Лакса для блочной якобиевой нормальной матрицы}

1. Пусть $J$ - блочная матрица вида (5), не обязательно нормальная. Предположим, что ее элементы непрерывно дифференцируемым образом зависят от $t \in[0, T](T \leqslant \infty): J=J(t)$. Рассмотрим произвольную бесконечномерную матрицу $A=\left(\alpha_{j, k}\right)_{j, k=0}^{\infty}, \alpha_{j, k}: \mathscr{H}_{k} \rightarrow \mathscr{H}_{j}$. Пусть ее матричные элементы $\alpha_{j, k}$ зависят от того же $t$ непрерывно: $\alpha_{j, k}=\alpha_{j, k}(t)$.

Рассмотрим уравнение Лакса с коммутатором $[\cdot, \cdot]$ :

$$
\left(\frac{d J}{d t}\right)(t)=: \dot{J}(t)=[J(t), A(t)]:=J(t) A(t)-A(t) J(T), \quad t \in[0, T] .
$$


Здесь произведение $J A$ блочных матриц $J=\left(a_{j, k}\right)_{j, k=0}^{\infty}$ и $A=\left(\alpha_{j, k}\right)_{j, k=0}^{\infty}$, $a_{j, k}, \alpha_{j, k}: \mathscr{H}_{k} \rightarrow \mathscr{H}_{j}$, определено обычным образом: $(j, k)$-й элемент $(J A)_{j, k}$ произведения подсчитывается по формуле

$$
(J A)_{j, k}=\sum_{l=0}^{\infty} a_{j, l} \alpha_{l, k}, \quad j, k \in \mathbb{N}_{0} .
$$

У нас $J$ всегда будет блочной якобиевой матрицей; поэтому в (18) вместо ряда стоит конечная сумма (максимум трех слагаемых).

Если в равенстве $(17)$ матрицу $A(t)$ считать фиксированной матрицей коэффициентов, то его можно воспринимать как линейное дифференциальное уравнение первого порядка относительно неизвестной $J(t), t \in[0, T]$. Однако если элементы $\alpha_{j, k}(t)$ матрицы $A(t)$ зависят каким-либо заданным способом от элементов $a_{j, k}(t)$ матрицы $J(t)$ (например, $\alpha_{j, k}(t)=a_{j, k}(t)$ для некоторых $j, k$ ), то равенство (17) превращается в некоторую систему дифференциальных уравнений первого порядка по $t$ с нелинейной правой частью. Хорошо известно ([2], [3]), что система Тоды образуется именно таким образом, с использованием в качестве $J(t)$ обычной самосопряженной якобиевой матрицы.

В этой статье будут приведены некоторые примеры таких систем и дан способ их интегрирования, основанный на спектральной теории блочных якобиевых нормальных матриц.

В дальнейшем существенную роль играет характер спектра нормального оператора $\boldsymbol{J}(t)$ : он расположен на одномерной кривой из $\mathbb{C}$ или заполняет некоторый компакт из $\mathbb{C}$, содержащий открытую область. Первому случаю посвящены $\S 3$ и $\S 4$, второму посвящен $\S 5$.

2. Выпишем уравнение Лакса (17) в форме уравнения для элементов матриц в случае, когда $A(t)$ имеет блочную якобиеву структуру, подобную структуре матрицы (5) $\left(n \in \mathbb{N}_{0}\right)$ :

$$
A(t)=\left(\begin{array}{cccccc}
\beta_{0} & \gamma_{0} & 0 & 0 & 0 & \ldots \\
\alpha_{0} & \beta_{1} & \gamma_{1} & 0 & 0 & \ldots \\
0 & \alpha_{1} & \beta_{2} & \gamma_{2} & 0 & \ldots \\
\vdots & \vdots & \vdots & \vdots & \vdots & \ddots
\end{array}\right), \quad \begin{aligned}
& \alpha_{n}: \mathscr{H}_{n} \rightarrow \mathscr{H}_{n+1}, \\
& \gamma_{n}: \mathscr{H}_{n} \rightarrow \mathscr{H}_{n+1} \rightarrow \mathscr{H}_{n} .
\end{aligned}
$$

Мы сейчас и в дальнейшем, как правило, не указываем явно зависимость элементов матрицы (19) от $t \in[0, T]$. То же самое относится и к матрице $J(t)$ : она будет записываться в виде (5). Сопряженная матрица $A^{+}(t)$ строится по тому же правилу, что и $J^{+}(t)$ (см. $\S 1$, разд. 2$)$.

Произведение двух трехдиагональных матриц будет пятидиагональной матрицей. Для подсчета коммутатора $[J(t), A(t)]$ (также пятидиагональной матрицы) отметим следующие формулы (в дальнейшем всюду элемент с отрицательным индексом считается равным нулю): для любого $n \in \mathbb{N}_{0}$

$$
\begin{aligned}
(J A)_{n-2, n} & =c_{n-2} \gamma_{n-1}, \\
(J A)_{n-1, n} & =b_{n-1} \gamma_{n-1}+c_{n-1} \beta_{n}, \\
(J A)_{n, n} & =a_{n-1} \gamma_{n-1}+b_{n} \beta_{n}+c_{n} \alpha_{n}, \\
(J A)_{n+1, n} & =a_{n} \beta_{n}+b_{n+1} \alpha_{n}, \\
(J A)_{n+2, n} & =a_{n+1} \alpha_{n} .
\end{aligned}
$$


Для $A J$ выполняются аналогичные формулы с заменой $a_{j}, b_{j}, c_{j}$ на $\alpha_{j}, \beta_{j}, \gamma_{j}$ соответственно и, наоборот, $\alpha_{j}, \beta_{j}, \gamma_{j}$ на $a_{j}, b_{j}, c_{j}$.

Сравнивая элементы в левой и правой частях уравнения (17) и пользуясь формулами (20), получим уравнение Лакса в «координатной» форме: для любого $n \in \mathbb{N}_{0}$

$$
\begin{aligned}
0 & =c_{n} \gamma_{n+1}-\gamma_{n} c_{n+1} \\
\dot{c}_{n} & =b_{n} \gamma_{n}+c_{n} \beta_{n+1}-\beta_{n} c_{n}-\gamma_{n} b_{n+1} \\
\dot{b}_{n} & =a_{n-1} \gamma_{n-1}+b_{n} \beta_{n}+c_{n} \alpha_{n}-\alpha_{n-1} c_{n-1}-\beta_{n} b_{n}-\gamma_{n} a_{n} \\
\dot{a}_{n} & =a_{n} \beta_{n}+b_{n+1} \alpha_{n}-\alpha_{n} b_{n}-\beta_{n+1} a_{n} \\
0 & =a_{n+1} \alpha_{n}-\alpha_{n+1} a_{n}
\end{aligned}
$$

Нормы элементов матрицы (19) будем также считать равномерно ограниченными по $n \in \mathbb{N}_{0}$ и $t \in[0, T]$. Поэтому она порождает в $\mathbf{l}_{2}$ ограниченный оператор, для которого мы сохраним обозначение $A(t)$. Оператор $A(t)$ будет слабо непрерывным по $t \in[0, T]$.

\section{§3. Случай одномерного спектра блочной нормальной якобиевой матрицы}

1. Пусть $J(t), t \in[0, T], T \leqslant \infty,-$ блочная нормальная матрица с непрерывно дифференцируемыми по $t$ коэффициентами. Нормы ее элементов предполагаются равномерно ограниченными по $j, k \in \mathbb{N}_{0}$ и $t \in[0, T]$. Таким образом, матрица $J(t)$ порождает в пространстве $\mathbf{l}_{2}$ ограниченный нормальный оператор $\boldsymbol{J}(t)$ с равномерно ограниченной по $t \in[0, T]$ нормой. Он будет слабо непрерывно дифференцируемым.

Предположим, что спектр оператора $\boldsymbol{J}(t)$ (содержащий $z$ и $\bar{z}$ одновременно) для любого $t$ расположен на конечной кривой $S$ из $\mathbb{C}$. Мы будем сейчас рассматривать общий случай, когда функции (10), суженные на $S$, остаются линейно независимыми. Разумеется, условие линейной независимости выполняется не всегда (см. §4).

Будем считать, что кривая $S$ является непрерывным образом фиксированного отрезка $[a, b] \subset \mathbb{R}, S=\{F(s) \in \mathbb{C}: s \in[a, b]\}$, где $F$ - некоторая непрерывная комплекснозначная функция на $[a, b]$. При этом предполагается, что $S$ не имеет петель, т. е. ее дополнение в $\mathbb{C}$ односвязно. С другой стороны, результаты сохраняются и для случая, когда $S$ гомотопна окружности.

В дальнейшем существенную роль будет играть функция Вейля оператора $\boldsymbol{J}(t)$

$$
m(z ; t)=\int_{S} \frac{d \rho(\zeta ; t)}{\zeta-z},
$$

определенная для всех регулярных точек $z$ оператора $\boldsymbol{J}(t)$ и тем более для всех $z \in \mathbb{C} \backslash S$. При фиксированном $t \in[0, T]$ функция $m(z ; t), z \in \mathbb{C} \backslash S$, однозначно определяет спектральную вероятностную меру $d \rho(\zeta ; t)$ (см., например, [40], [41], а также замечания 1,2 в 5 ).

Пусть $R_{z}(t)$ - резольвента оператора $\boldsymbol{J}(t), t \in[0, T]$, являющаяся ограниченным оператором в пространстве $\mathbf{l}_{2}$. Как всякий ограниченный оператор в $\mathbf{l}_{2}$, 
она изображается операторной матрицей $\left(r_{j, k}(z ; t)\right)_{j, k=0}^{\infty}$ :

$$
\begin{gathered}
\left(R_{z}(t) f\right)_{j}=\sum_{k=0}^{\infty} r_{j, k}(z ; t) f_{k}, \quad r_{j, k}(z, t): \mathscr{H}_{k} \rightarrow \mathscr{H}_{j}, \\
\left(r_{j, k}(z ; t)\right)_{\alpha, \beta}=\left(r_{j, k}(z ; t) \delta_{k ; \beta}, \delta_{j ; \alpha}\right)_{\alpha, \beta=0}^{j, k}
\end{gathered}
$$

для любого $f \in \mathbf{l}_{2}$. Здесь $\delta_{n ; \alpha}, \alpha=0, \ldots, n,-$ ортонормированный стандартный базис в пространстве $\mathscr{H}_{n}=\mathbb{C}^{n+1}, n \in \mathbb{N}_{0}$ (см. $\S 1$, разд. 2).

Нам понадобятся начальные элементы этой матрицы, а именно

$$
\begin{gathered}
r_{0,0}(z ; t)=\left(R_{z}(t) \delta_{0}, \delta_{0}\right)_{\mathbf{l}_{2}}=\int_{S} \frac{d \rho(\zeta ; t)}{\zeta-z}=m(z ; t), \\
r_{1,0}(z ; t)=\left(\begin{array}{c}
\left(R_{z}(t) \delta_{0}, \delta_{1 ; 0}\right)_{\mathbf{l}_{2}} \\
\left(R_{z}(t) \delta_{0}, \delta_{1 ; 1}\right)_{\mathbf{l}_{2}}
\end{array}\right), \\
r_{0,1}(z ; t)=\left(\left(R_{z}(t) \delta_{1 ; 0}, \delta_{0}\right)_{\mathbf{l}_{2}} \quad\left(R_{z}(t) \delta_{1 ; 1}, \delta_{0}\right)_{\mathbf{l}_{2}}\right) .
\end{gathered}
$$

Дифференцируемость по $t$ элементов матрицы $J(t)$ вызывает слабую дифференцируемость оператора $\boldsymbol{J}(t)$ и его резольвенты $R_{z}(t)$, а значит, и функции $m(z ; t)$. Кроме того, как легко проверить, для $z \in \mathbb{C} \backslash S$

$$
\begin{gathered}
(\boldsymbol{J}(t)-z \mathrm{Id})^{\cdot}=[\boldsymbol{J}(t)-z \mathrm{Id}, A(t)], \\
\dot{R}_{z}(t)=-R_{z}(t)(\boldsymbol{J}(t)-z \mathrm{Id})^{\cdot} \cdot R_{z}(t)=\left[R_{z}(t), A(t)\right], \quad t \in[0, T] .
\end{gathered}
$$

Лемма 1. Справедливо представление

$$
\begin{gathered}
\dot{m}(z ; t)=\alpha_{0 ; 0,0}\left(R_{z}(t) \delta_{1 ; 0}, \delta_{0}\right)_{\mathbf{l}_{2}}+\alpha_{0 ; 1,0}\left(R_{z}(t) \delta_{1 ; 1}, \delta_{0}\right)_{\mathbf{l}_{2}}-\gamma_{0 ; 0,0}\left(R_{z}(t) \delta_{0}, \delta_{1 ; 0}\right)_{\mathbf{l}_{2}} \\
-\gamma_{0 ; 0,1}\left(R_{z}(t) \delta_{0}, \delta_{1,1}\right)_{\mathbf{l}_{2}}, \quad z \in \mathbb{C} \backslash S, t \in[0, T] .
\end{gathered}
$$

Напомним, что оператор $D: \mathscr{H}_{m} \rightarrow \mathscr{H}_{n}, m, n \in \mathbb{N}_{0}$, задается в нашем стандартном базисе матрицей $\left(d_{\alpha, \beta}\right)_{\alpha, \beta=0}^{n, m}$, где $d_{\alpha, \beta}=\left(D \delta_{m ; \beta}, \delta_{n ; \alpha}\right) \mathscr{H}_{n}$.

Доказательство. На основании формул (25), (24) и (19) имеем

$$
\begin{aligned}
\dot{m}(z ; t)= & \left(R_{z}(t) \delta_{0}, \delta_{0}\right)_{\mathbf{l}_{2}}=\left(\dot{R}_{z}(t) \delta_{0}, \delta_{0}\right)_{\mathbf{l}_{2}}=\left(\left(R_{z}(t) A(t)-A(t) R_{z}(t)\right) \delta_{0}, \delta_{0}\right)_{\mathbf{l}_{2}} \\
= & \left(R_{z}(t) A(t) \delta_{0}, \delta_{0}\right)_{\mathbf{l}_{2}}-\left(A(t) R_{z}(t) \delta_{0}, \delta_{0}\right)_{\mathbf{l}_{2}} \\
= & \left(\delta_{0}, A^{*}(t) R_{z}^{*}(t) \delta_{0}\right)_{\mathbf{l}_{2}}-\left(A(t) R_{z}(t) \delta_{0}, \delta_{0}\right)_{\mathbf{l}_{2}} \\
= & \frac{\left(A^{*}(t) R_{z}^{*}(t) \delta_{0}, \delta_{0}\right)_{\mathbf{l}_{2}}}{-\left(A(t) R_{z}(t) \delta_{0}, \delta_{0}\right)_{\mathbf{l}_{2}}} \\
= & \frac{\left(A^{*}(t)\left[\left(R_{z}^{*}(t) \delta_{0}, \delta_{0}\right)_{\mathbf{l}_{2}},\left(R_{z}^{*}(t) \delta_{0}, \delta_{1 ; 0}\right)_{\mathbf{l}_{2}},\left(R_{z}^{*}(t) \delta_{0}, \delta_{1 ; 1}\right)_{\mathbf{l}_{2}}, \ldots\right], \delta_{0}\right)_{\mathbf{l}_{2}}}{} \\
& \quad-\left(A(t)\left[\left(R_{z}(t) \delta_{0}, \delta_{0}\right)_{\mathbf{l}_{2}},\left(R_{z}(t) \delta_{0}, \delta_{1 ; 0}\right)_{\mathbf{l}_{2}},\left(R_{z}(t) \delta_{0}, \delta_{1 ; 1}\right)_{\mathbf{l}_{2}}, \ldots\right], \delta_{0}\right)_{\mathbf{l}_{2}} \\
= & \beta_{0}\left(R_{z}(t) \delta_{0}, \delta_{0}\right)_{\mathbf{l}_{2}}+\alpha_{0 ; 0,0}\left(R_{z}(t) \delta_{1 ; 0}, \delta_{0}\right)_{\mathbf{l}_{2}}+\alpha_{0 ; 1,0}\left(R_{z}(t) \delta_{1 ; 1}, \delta_{0}\right)_{\mathbf{l}_{2}} \\
& \quad-\beta_{0}\left(R_{z}(t) \delta_{0}, \delta_{0}\right)_{\mathbf{l}_{2}}-\gamma_{0 ; 0,0}\left(R_{z}(t) \delta_{0}, \delta_{1 ; 0}\right)_{\mathbf{l}_{2}}-\gamma_{0 ; 0,1}\left(R_{z}(t) \delta_{0}, \delta_{1 ; 1}\right)_{\mathbf{l}_{2}} .
\end{aligned}
$$

После сокращения 1-го и 4-го слагаемых в (27) мы придем к (26).

Лемма 2. Справедливо представление

$$
\begin{gathered}
c_{0 ; 0,0}\left(R_{z}(t) \delta_{0}, \delta_{1 ; 0}\right)_{\mathbf{l}_{2}}+c_{0 ; 0,1}\left(R_{z}(t) \delta_{0}, \delta_{1 ; 1}\right)_{\mathbf{l}_{2}}=\left(z-b_{0}\right) m(z ; t)+1, \\
z \in \mathbb{C} \backslash S, t \in[0, T] .
\end{gathered}
$$


Доказательство. Из равенства $(J(t)-z \mathrm{Id}) R_{z}(t)=\mathrm{Id}$ следует, что

$$
\left(J(t) R_{z}(t) \delta_{0}, \delta_{0}\right)_{\mathbf{l}_{2}}=z m(z ; t)+1, \quad z \in \mathbb{C} \backslash S, t \in[0, T] .
$$

Но, согласно (5),

$$
\begin{aligned}
z m(z ; t)+1 & =\left(J(t) R_{z}(t) \delta_{0}, \delta_{0}\right)_{\mathbf{l}_{2}} \\
& =\left(J(t)\left[\left(R_{z}(t) \delta_{0}, \delta_{0}\right)_{\mathbf{l}_{2}},\left(R_{z}(t) \delta_{0}, \delta_{1 ; 0}\right)_{\mathbf{l}_{2}},\left(R_{z}(t) \delta_{0}, \delta_{1,1}\right)_{\mathbf{l}_{2}}, \ldots\right], \delta_{0}\right)_{\mathbf{l}_{2}} \\
& =b_{0} m(z ; t)+c_{0 ; 0,0}\left(R_{z}(t) \delta_{0}, \delta_{1 ; 0}\right)_{\mathbf{l}_{2}}+c_{0 ; 0,1}\left(R_{z}(t) \delta_{0}, \delta_{1 ; 1}\right)_{\mathbf{l}_{2}} .
\end{aligned}
$$

Теорема 3. Пусть рассматриваемая блочная нормальная матрица $J(t)$ удовлетворяет уравнению Лакса (21) с матрищей $A(t)$ вида (19), у которой

$$
\alpha_{0}(t)=0 \quad u \quad \gamma_{0}(t)=-c_{0}(t), \quad t \in[0, T] .
$$

Тогда функиия Вейля $m(z ; t)$, построенная по нормальному оператору $\boldsymbol{J}(t)$, удовлетворяет уравнению

$$
\dot{m}(z ; t)=\left(z-b_{0}(t)\right) m(z ; t)+1, \quad z \in \mathbb{C} \backslash S, t \in[0, T] .
$$

Спектральная мера этого оператора имеет вид

$$
d \rho(\zeta ; t)=C(t) e^{\zeta t} d \rho(\zeta ; 0), \quad \zeta \in S, t \in[0, T] .
$$

Здесь $C(t)>0$ - множитель, нормирующий меру (31) к вероятностной, т.е. долэнно бытъ $\rho(S ; t)=1$ для любого $t \in[0, T]$.

Отметим, что равенство (31) влечет за собой изоспектральность операторной функции $\boldsymbol{J}(t), t \in[0, T]:$ спектр оператора $\boldsymbol{J}(t)$ не зависит от $t$.

Доказательство. Равенства (29), (26) и (28) дают

$$
\begin{aligned}
\dot{m}(z ; t) & =c_{0 ; 0,0}\left(R_{z}(t) \delta_{0}, \delta_{1 ; 0}\right)_{\mathbf{l}_{2}}+c_{0 ; 0,1}\left(R_{z}(t) \delta_{0}, \delta_{1 ; 1}\right)_{\mathbf{l}_{2}} \\
& =\left(z-b_{0}(t)\right) m(z ; t)+1, \quad z \in \mathbb{C} \backslash S, t \in[0, T] .
\end{aligned}
$$

Установим эквивалентность соотношений (30) и (31). Дифференцируемость по $t$ функции $m(z ; t)$ эквивалентна дифференцируемости функции $J(t)-z \operatorname{Id}$ и дифференцируемости меры $d \rho(\zeta ; t)$ (т. е. функции $\rho(\Delta ; t)$ для любого борелевского множества $\Delta \subset S$ ) (см., например, [40], [41], а также замечания 1,2 в $\S 5)$. Поэтому, согласно условию (29) и тому, что спектральная мера является вероятностной, можно написать

$$
\begin{aligned}
\int_{S} \frac{d \dot{\rho}(\zeta ; t)}{\zeta-z} & =\frac{d}{d t}\left(\int_{S} \frac{d \rho(\zeta ; t)}{\zeta-z}\right)=\dot{m}(z ; t)=\left(z-b_{0}(t)\right) m(z ; t)+1 \\
& =\int_{S} \frac{\left(z-b_{0}(t)\right)+(\zeta-z)}{\zeta-z} d \rho(\zeta ; t) \\
& =\int_{S} \frac{\zeta-b_{0}(t)}{\zeta-z} d \rho(\zeta ; t), \quad z \in \mathbb{C} \backslash S, t \in[0, T] .
\end{aligned}
$$

Отсюда следует, что $d \dot{\rho}(\zeta ; t)=\left(\zeta-b_{0}(t)\right) d \rho(\zeta ; t)$. Интегрируя это уравнение относительно $t$, получаем (31).

3. Фиксируя теперь в уравнении Лакса $(21)$ матрицу $A(t)$ тем или иным образом (ее элементы зависят от элементов матрицы $J(t)$ ), но с соблюдением условий (29), мы получим дифференциальное уравнение по $t$ относительно 
$a_{n}(t), b_{n}(t)$ и $c_{n}(t)$ первого порядка с нелинейной правой частью. Если это уравнение имеет решение, для которого матрица $J(t)$ вида (5) будет нормальной, то соответствующая спектральная мера задается формулой (31).

Мы ограничимся здесь наиболее простым подобным выбором $A(t)$. А именно, положим

$$
\alpha_{n}(t)=0, \quad \beta_{n}(t)=-\frac{1}{2} b_{n}(t), \quad \gamma_{n}(t)=-c_{n}(t), \quad n \in \mathbb{N}_{0}, t \in[0, T] .
$$

Условие (29) будет выполнено. Рассмотрим уравнение Лакса (21). Первое и последнее уравнения, очевидно, выполняются. Остальные принимают вид известной системы типа Полякова

$$
\begin{array}{rlrl}
\dot{a}_{n} & =\frac{1}{2}\left(b_{n+1} a_{n}-a_{n} b_{n}\right), & & a_{n}: \mathscr{H}_{n} \rightarrow \mathscr{H}_{n+1}, \\
\dot{b}_{n}=c_{n} a_{n}-a_{n-1} c_{n-1}, & b_{n}: \mathscr{H}_{n} \rightarrow \mathscr{H}_{n}, \\
\dot{c}_{n}=\frac{1}{2}\left(c_{n} b_{n+1}-b_{n} c_{n}\right), & c_{n}: \mathscr{H}_{n+1} \rightarrow \mathscr{H}_{n} .
\end{array}
$$

Здесь $t \in[0, T], T \leqslant \infty, a_{-1}=c_{-1}=0, n \in \mathbb{N}_{0}$.

Покажем, что задачу Коши для системы (33) можно решить подобно случаю цепочки Тоды, но с привлечением спектральной теории блочных нормальных якобиевых матриц вместо обычных якобиевых.

Теорема 4. Рассмотрим систему (33), где $a_{n}(t), b_{n}(t) u c_{n}(t), t \in[0, T]$, $n \in \mathbb{N}_{0}$, - равномерно по $n$ u $t$ ограниченные по норме и непрерывно дифберениируемые по $t$ матричные функиии, причем $a_{n}(t), c_{n}(t)$ имеют структуру $(7)$. Поставим для нее задачу Коши: по начальным данным $\left(a_{n}(0)\right)_{n=0}^{\infty},\left(b_{n}(0)\right)_{n=0}^{\infty}$, $\left(c_{n}(0)\right)_{n=0}^{\infty}$ найти решение системь $(33)$ на $[0, T]$.

Предположим, что блочная якобиева матрица $J(0)$, построенная в соответствии с формулой (5) по начальным данным, порожсдает нормальный ограниченный оператор $\boldsymbol{J}(0)$ в пространстве $\mathbf{1}_{2}$, спектр которого (не сводящийся $\kappa$ конечному множеству) расположен на непрерывной кривой $S$ в комплексной плоскости $\mathbb{C}$, содержкащей вместе с точкой z точку $\bar{z}$ и такой, что на ней функиии (10) линейно независимь.

Тогда решение $\left(a_{n}(t)\right)_{n=0}^{\infty},\left(b_{n}(t)\right)_{n=0}^{\infty},\left(c_{n}(t)\right)_{n=0}^{\infty}, t \in[0, T]$, этой задачи сушествует и образует, в соответствии с (5), якобиеву блочную нормальную матрицу $J(t)$. Спектральная мера $d \rho(\zeta ; t)$ соответствующего оператора $\boldsymbol{J}(t)$ сосредоточена на $S$ и задается формулой $(31)$, где $d \rho(\zeta ; 0)$ - спектральная мера оператора $\boldsymbol{J}(0)$.

Само решение восстанавливается по мере $d \rho(\zeta ; t)$, согласно теореме 2 , при помощи формуль (16).

Доказательство. По вероятностной мере $d \rho(\zeta ; t)$ при помощи процедуры ортогонализации системы (10) строится система (11) ортонормированных полиномов от $z$ и $\bar{z}$, зависящих от времени $t \in[0, T]$. При помощи (16) находятся элементы $a_{n}(t), b_{n}(t), c_{n}(t)$ якобиевой матрицы $J(t)$. Затем проверяется, что они удовлетворяют системе (33).

Так, проверим, что удовлетворяется первое из уравнений (33). Остальные два проверяются аналогично. Прежде всего, включим $C(t)$ из $(31)$ в выражения для $P_{n ; \alpha}(z)$ из $(11)$ : каждый столбец в $(11)$ умножим на $(C(t))^{1 / 2}$. Тогда новые векторы (8) по-прежнему будут компонентами обобщенного собственного 
вектора $P(z)$ и уравнения $(9)$ сохраняются. Формула для $a_{n}$, согласно $(16)$ и (31), приобретает вид

$$
\begin{gathered}
a_{n ; \alpha, \beta}(t)=a_{n+1, n ; \alpha, \beta}(t)=\int_{S} \zeta \overline{P_{n ; \beta}(\zeta ; t)} P_{n+1 ; \alpha}(\zeta ; t) e^{\zeta t} d \rho(\zeta ; 0) \quad \forall n \in \mathbb{N}_{0}, \\
\alpha=0, \ldots, n+1, \quad \beta=0, \ldots, n
\end{gathered}
$$

(мы указываем зависимость полиномов от $t$ : ортогонализация при построении системы (11) ведется относительно меры $d \rho(\zeta ; t)=e^{\zeta t} d \rho(\zeta ; 0)$ для каждого $t \in$ $[0, T])$.

Дифференцируя (34) по $t$ под знаком интеграла, получим

$$
\begin{aligned}
\dot{a}_{n ; \alpha, \beta}(t)=\int_{S}\left(\zeta \overline{\dot{P}_{n ; \beta}(\zeta ; t)} P_{n+1 ; \alpha}(\zeta ; t)\right. & +\zeta \overline{P_{n ; \beta}(\zeta ; t)} \dot{P}_{n+1 ; \alpha}(\zeta ; t) \\
& \left.+\zeta^{2} \overline{P_{n ; \beta}(\zeta ; t)} P_{n+1 ; \alpha}(\zeta ; t)\right) e^{\zeta t} d \rho(\zeta ; 0) .
\end{aligned}
$$

Подсчитаем вклад в $\dot{a}_{n}$ третьего слагаемого в (35). Для этого воспользуемся первым и вторым из равенств (9) (при $z=\zeta$ и $\bar{z}=\bar{\zeta}$ ), которым удовлетворяет вектор $\overline{P_{n ; \cdot}(\zeta ; t)}$. В результате вместо этого слагаемого получим сумму девяти слагаемых, содержащих произведения $\overline{P_{j ; \cdot}(\zeta ; t)} P_{k ; \cdot}(\zeta ; t)$ с операторными коэффициентами - элементами матриц $J(t)$ и $J^{+}(t)$. Учитывая ортономированность системы (11), после несложных подсчетов получим, что искомый вклад равен $\left(a_{n} b_{n}\right)_{\alpha, \beta}+\left(b_{n+1} a_{n}\right)_{\alpha, \beta}$.

Найдем вклад первого слагаемого в (35). Для этого продифференцируем по $t$ второе из равенств (9) (при $\bar{z}=\bar{\zeta}$ ). В результате получим выражение для $\overline{\bar{\zeta} \dot{P}_{n ; \beta}(\zeta ; t)}$ в виде шести слагаемых, состоящих из $\overline{P_{j ;} \cdot(\zeta ; t)}, j=n-1, n, n+1$, и их первых производных по $t$ с операторными коэффициентами - элементами матриц $J^{+}(t)$ и $\dot{J}^{+}(t)$. Вклад этого слагаемого равен $\dot{a}_{n ; \alpha, \beta}-\frac{1}{2}\left(b_{n+1} a_{n}\right)_{\alpha, \beta}$.

При этом нужно воспользоваться ортонормированностью системы (11) и равенством

$$
\int_{S} \overline{\dot{P}_{n+1 ; \beta}(\zeta ; t)} P_{n+1 ; \alpha}(\zeta ; t) e^{\zeta t} d \rho(\zeta ; 0)=-\frac{1}{2} b_{n+1 ; \alpha, \beta},
$$

которое получается дифференцированием по $t$ соотношения

$$
\int_{S} \overline{P_{n+1 ; \beta}(\zeta ; t)} P_{n+1 ; \alpha}(\zeta ; t) e^{\zeta t} d \rho(\zeta ; 0)=\delta_{\alpha, \beta}, \quad \alpha, \beta=0, \ldots, n+1 .
$$

Аналогично подсчитывается вклад второго слагаемого в (35). При этом нужно продифференцировать по $t$ первое из равенств (9) при $z=\zeta$ с заменой $n$ на $n+1$. Сейчас опять используется равенство (36) при $n$ вместо $n+1$ и аналогичное ему соотношение для интеграла от $\overline{P_{n ; \beta}(\zeta ; t)} \dot{P}_{n+1 ; \alpha}(\zeta ; t)$ (он равен $\left.-a_{n ; \alpha, \beta}\right)$, которое получается дифференцированием (37) с $n ; \beta$ вместо $n+1 ; \beta$. Вклад второго слагаемого равен $\dot{a}_{n ; \alpha, \beta}-\frac{1}{2}\left(a_{n} b_{n}\right)_{\alpha, \beta}-\left(b_{n+1} a_{n}\right)_{\alpha, \beta}$.

В конечном счете (35) дает

$$
\dot{a}_{n}=\left(a_{n} b_{n}+b_{n+1} a_{n}\right)+\left(\dot{a}_{n}-\frac{1}{2} b_{n+1} a_{n}\right)+\left(\dot{a}_{n}-\frac{1}{2} a_{n} b_{n}-b_{n+1} a_{n}\right),
$$

т. е. первое из уравнений (33). 


\section{§4. Случай унитарной блочной якобиевой матрицы}

1. В случае когда $J$ - унитарная блочная якобиева матрица в пространстве $\mathbf{l}_{2}$, кривая $S$ превращается в единичную окружность $\mathbb{T}=\{z \in \mathbb{C}:|z|=1\}$ и функции (10) становятся линейно зависимыми. Согласно [7], [8], соответствующий матрице $J$ оператор следует рассматривать не во всем пространстве $\mathbf{l}_{2}$, a в его подпространстве $\mathbf{l}_{2, u}$, имеющем вид

$$
\mathrm{l}_{2, u}=\mathscr{H}_{0} \oplus \mathscr{H}_{1} \oplus \mathscr{H}_{2} \oplus \cdots, \quad \text { где } \mathscr{H}_{0}=\mathbb{C}, \mathscr{H}_{1}=\mathscr{H}_{2}=\cdots=\mathbb{C}^{2} \text {. }
$$

Блоки унитарной якобиевой матрицы $(5)$, действующей в $\mathrm{l}_{2, u}$, имеют вид

$$
\begin{gathered}
a_{0}=\left(\begin{array}{c}
a_{0 ; 0,0} \\
0
\end{array}\right), \quad b_{0}=\left(\begin{array}{l}
b_{0 ; 0,0}
\end{array}\right), \quad c_{0}=\left(\begin{array}{ll}
c_{0 ; 0,0} & c_{0 ; 0,1}
\end{array}\right), \\
a_{n}=\left(\begin{array}{cc}
a_{n ; 0,0} & a_{n ; 0,1} \\
0 & 0
\end{array}\right), \quad b_{n}=\left(\begin{array}{cc}
b_{n ; 0,0} & b_{n ; 0,1} \\
b_{n ; 1,0} & b_{n ; 1,1}
\end{array}\right), \quad c_{n}=\left(\begin{array}{cc}
0 & 0 \\
c_{n ; 1,0} & c_{n ; 1,1}
\end{array}\right), \\
a_{0 ; 0,0}, c_{0 ; 0,1}, a_{n ; 0,0}, c_{n ; 1,1}>0, \quad n \in \mathbb{N} .
\end{gathered}
$$

Элементы этих блоков зависят определенным образом от последовательности комплексных чисел $\left(v_{n}\right)_{n=0}^{\infty},\left|v_{n}\right|<1$, - коэффициентов Верблунского, обеспечивающих унитарность оператора $J$ в $\mathbf{l}_{2, u}$ (см. [36]-[38]).

Сейчас вместо последовательности (10) нужно ортогонализировать ее часть

$$
z^{0} \bar{z}^{0}=1 ; z^{1} \bar{z}^{0}=z^{1}, z^{0} \bar{z}^{1}=\bar{z}^{1} ; \ldots ; z^{n} \bar{z}^{0}=z^{n}, z^{0} \bar{z}^{n}=\bar{z}^{n} ; \ldots, \quad z \in \mathbb{T} .
$$

В результате получим систему ортогональных полиномов (11), в которой в каждом столбце, начиная со второго, будут фигурировать только два полинома; их удобно обозначать через $P_{n ; 0}(z), P_{n ; 1}(z), n \in \mathbb{N}$. Имеет место соответствующая модификация теорем 1, 2 (см. подробнее [7], [8]).

Рассмотрим уравнение Лакса (17), в котором $J(t)$ и $A(t), t \in[0, T],-$ блочные якобиевы матрицы вида (5) и (19), но действуют они не в пространстве $\mathbf{l}_{2}$, а в его подпространстве $\mathbf{l}_{2, u}$ вида (38). Таким образом, все матрицы $a_{n}, b_{n}, c_{n}, \alpha_{n}$, $\beta_{n}, \gamma_{n}$ при $n \in \mathbb{N}$ являются квадратными матрицами размера $2 \times 2$, зависящими от $t$. При $n=0$ матрицы $a_{0}, b_{0}, c_{0}$ имеют вид $(39)$, а $\alpha_{0}, \beta_{0}, \gamma_{0}$ - произвольные матрицы соответствующей размерности. Предполагается, что $a_{n}, c_{n}$ имеют структуру (40) и матрица $J(t)$ унитарна при каждом $t$.

Легко понять, что уравнение Лакса и сейчас запишется в той же координатной форме (21), но с операторами, действующими между пространствами (38). Результаты §3, разд. 1, не изменятся, только роль $S$ будет играть единичная окружность $S=\mathbb{T}$. Леммы 1 и 2 также сохранятся в прежнем виде: в их формулировках и доказательствах фигурируют только элементы матриц $\alpha_{0}, \beta_{0}, \gamma_{0}$, $b_{0}, c_{0}$ и характер разложения векторов $R_{z}(t) \delta_{0}$ и $R_{z}^{*}(t) \delta_{0}$ по базисным векторам $\delta_{0} ; \delta_{1 ; 0}, \delta_{1 ; 1} ; \ldots$, а они такие же, как и в $\S 3$. Таким образом, справедлив и аналог теоремы 3: условия (29), уравнение (30) и представление (31) для спектральной меры будут прежними.

Теорема 4 также сохраняется. Так, нужно рассмотреть систему (33), в которой пространства $\mathscr{H}_{n}$ имеют вид не (4), а (38), и начальная матрица $J(0)$ является унитарной в $\mathbf{l}_{2, u}$, а не произвольной нормальной в $\mathbf{l}_{2}$ матрицей. Восстановление решения $\left(a_{n}(t)\right)_{n=0}^{\infty},\left(b_{n}(t)\right)_{n=0}^{\infty},\left(c_{n}(t)\right)_{n=0}^{\infty}$ по начальным данным, образующим $J(0)$, т. е. по $\left(a_{n}(0)\right)_{n=0}^{\infty},\left(b_{n}(0)\right)_{n=0}^{\infty},\left(c_{n}(0)\right)_{n=0}^{\infty}$, производится прежней процедурой: по начальной спектральной мере $d \rho(\zeta ; 0), \zeta \in \mathbb{T}$, построим меру 
$d \rho(\zeta ; t), \zeta \in \mathbb{T}, t \in[0, T]$, согласно формуле $(31)$, затем строим соответствующие ортогональные полиномы по этой мере $d \rho(\zeta ; t)$ и применяем формулу $(16)$. Доказательство сформулированного результата - повторение доказательства теоремы 4 .

В связи с конструкциями этого параграфа важную роль играют условия, обеспечивающие унитарность в пространстве $\mathbf{l}_{2, u}$ блочной якобиевой матрицы $J$ вида (5) с блоками (39), (40). Здесь имеется следующий красивый результат (см. [38], [36], [37]). По заданной произвольной последовательности комплексных чисел $\left(v_{n}\right)_{n=0}^{\infty},\left|v_{n}\right|<1$ (коэффициентам Верблунского), строим последовательность $2 \times 2$-матриц

$$
\Theta\left(v_{n}\right)=\left(\begin{array}{cc}
\bar{v}_{n} & \left(1-\left|v_{n}\right|^{2}\right)^{1 / 2} \\
\left(1-\left|v_{n}\right|^{2}\right)^{1 / 2} & -v_{n}
\end{array}\right), \quad n \in \mathbb{N}_{0},
$$

и их ортогональные суммы в обычном пространстве $\ell_{2}$ :

$$
\begin{gathered}
L=\Theta\left(v_{0}\right) \oplus \Theta\left(v_{2}\right) \oplus \Theta\left(v_{4}\right) \oplus \cdots \oplus \Theta\left(v_{2 n}\right) \oplus \cdots, \\
M=\mathbf{1} \oplus \Theta\left(v_{1}\right) \oplus \Theta\left(v_{3}\right) \oplus \Theta\left(v_{5}\right) \oplus \cdots \oplus \Theta\left(v_{2 n+1}\right) \oplus \cdots, \quad n \in \mathbb{N}_{0}
\end{gathered}
$$

(здесь $\mathbf{1}$ - единичный оператор в одномерном пространстве $\mathbb{C}$ ). Тогда матрица $J$ вида (5) с блоками (39), (40), понимаемая как обычная бесконечная матрица в пространстве $\ell_{2}$, будет унитарной тогда и только тогда, когда она имеет вид

$$
J=L M \text {. }
$$

Отметим, что результаты, подобные описанным выше в этом параграфе, были ранее получены в [39]. В этой работе рассматривались унитарные зависящие от времени пятидиагональные матрицы $J(t)$ структуры $(5),(39),(40),(42)$ в обычном пространстве $\ell_{2}$. Интегрировалось уравнение Лакса (17) с отличной от (32) матрицей $A(t)$. Оно сейчас совпадает с потоком Шура относительно переменных $\left(v_{n}(t)\right)_{n=0}^{\infty}$ вместо $\left(a_{n}(t)\right)_{n=0}^{\infty},\left(b_{n}(t)\right)_{n=0}^{\infty},\left(c_{n}(t)\right)_{n=0}^{\infty}$.

2. В заключение этого параграфа отметим, что легко привести примеры кривых $S$, отличных от $\mathbb{T}$ и от $[a, b] \subset \mathbb{R}$, содержащих $z \in \mathbb{C}$ и $\bar{z}$ одновременно, для которых функции (10) будут линейно зависимы. Такими будут, например, «половина $\mathbb{T} »$, т. е. $S=\{z \in \mathbb{T}: \operatorname{Re} z \geqslant 0\}$, вертикальный отрезок $S=\{z \in \mathbb{C}: \operatorname{Re} z=0,|\operatorname{Im} z| \leqslant 1\}$ (в последнем случае в каждой группе $z^{n} \bar{z}^{0}, z^{n-1} \bar{z}^{1}, \ldots, z^{0} \bar{z}^{n}$ при $n \geqslant 2$ будут встречаться одинаковые функции на $\left.S\right)$, И Т. П.

\section{§5. Случай двумерного спектра блочной нормальной якобиевой матрицы}

1. Пусть $J(t), t \in[0, T]$, - такая же, как и в $\S 3$, блочная нормальная зависящая от времени $t$ якобиева матрица, лишь с тем отличием, что ее спектр для любого $t$ является компактным множеством из $\mathbb{C}$, содержащим открытые подмножества. Пусть $S$ - замыкание ограниченной области из $\mathbb{C}$, содержащей спектр оператора $\boldsymbol{J}(t)$ для любого $t$, инвариантное относительно перехода $z \mapsto \bar{z}$. Можно считать, что спектральная мера $d \rho(\zeta ; t)$ - борелевская вероятностная мера на $S$. 
Сейчас построения будут несколько отличаться от проведенных в $\S 33,4$. Роль функции Вейля $m(z ; t)=\left(R_{z}(t) \delta_{0}, \delta_{0}\right)_{\mathbf{l}_{2}}$ (см. $\left.(22)\right)$ будет играть функция

$$
\begin{aligned}
M(z ; t): & =\left(R_{z}(t) \delta_{0}, R_{z}(t) \delta_{0}\right)_{\mathbf{l}_{2}}=\left(R_{z}^{*}(t) R_{z}(t) \delta_{0}, \delta_{0}\right)_{\mathbf{l}_{2}} \\
& =\int_{S} \frac{d \rho(\zeta ; t)}{(\zeta-z)(\bar{\zeta}-\bar{z})}, \quad z \in \mathbb{C} \backslash S, t \in[0, T] .
\end{aligned}
$$

Справедлива следующая общая теорема.

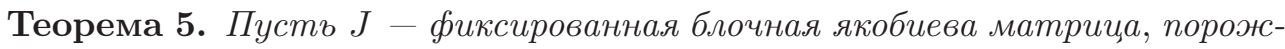
дающая в $\mathbf{l}_{2}$ нормальный ограниченный оператор $\boldsymbol{J}$. Пусть $R_{z}$ u $S$ - его резольвента и спектр. Тогда функиия $M(z):=\left(R_{z} \delta_{0}, R_{z} \delta_{0}\right)_{\mathbf{l}_{2}}, z \in \mathbb{C} \backslash S$, однозначно определяет спектральную меру $d \rho(\zeta), \zeta \in S$, этого оператора.

Доказательство. Функция $M(z)$ на основании спектральной теоремы допускает представление

$$
M(z)=\left(R_{z} \delta_{0}, R_{z} \delta_{0}\right)_{\mathbf{l}_{2}}=\int_{S} \frac{d \rho(\zeta)}{(\zeta-z)(\bar{\zeta}-\bar{z})} .
$$

Так как спектр $S$ расположен на круге $|z| \leqslant\|\boldsymbol{J}\|$, то при $|z|>\|\boldsymbol{J}\|$ функцию (44) можно записать в виде

$$
\begin{aligned}
M(z)= & \int_{S} \frac{d \rho(\zeta)}{(\zeta-z)(\bar{\zeta}-\bar{z})}=\frac{1}{|z|^{2}} \int_{S} \frac{d \rho(\zeta)}{\left(1-\zeta z^{-1}\right)\left(1-\bar{\zeta} \bar{z}^{-1}\right)} \\
= & \frac{1}{|z|^{2}} \int_{S} \sum_{m=0}^{\infty}\left(\frac{\zeta}{z}\right)^{m} \sum_{n=0}^{\infty}\left(\frac{\bar{\zeta}}{\bar{z}}\right)^{n} d \rho(\zeta) \\
= & \frac{1}{|z|^{2}} \int_{S} \sum_{m, n=0}^{\infty} \frac{1}{z^{m} \bar{z}^{n}} \zeta^{m} \bar{\zeta}^{n} d \rho(\zeta)=\frac{1}{|z|^{2}} \sum_{m, n=0}^{\infty} \frac{s_{m, n}}{z^{m} \bar{z}^{n}}, \\
& s_{m, n}=\int_{S} \zeta^{m} \bar{\zeta}^{n} d \rho(\zeta), \quad m, n \in \mathbb{N}_{0} .
\end{aligned}
$$

Из представления (45) функции $M(z)$ в виде ряда по $z^{-m} \bar{z}^{-n}$ при $|z|>\|\boldsymbol{J}\|$ и формулы Тейлора следует, что коэффициенты $s_{m, n}$ этого ряда по $M(z)$ восстанавливаются однозначно. Но эти коэффициенты являются (комплексными) моментами меры $d \rho(\zeta)$, допускающими оценку $\left|s_{m, n}\right| \leqslant\|\boldsymbol{J}\|^{m+n}, m, n \in \mathbb{N}_{0}$. Из теории комплексной проблемы моментов (см., например, [8], [42]) вытекает, что в этом случае мера $d \rho(\zeta)$ по $\left(s_{m, n}\right)_{m, n=0}^{\infty}$, а значит, и по $M(z)$ восстанавливается однозначно.

Таким образом, по функции (43) спектральная мера $d \rho(\zeta ; t), \zeta \in S$, при каждом $t \in[0, T]$ восстанавливается однозначно.

Сделаем два замечания в связи с теоремой 5 и ее доказательством.

Замечание 1. Пусть $S$ - ограниченная кривая описанного в начале $\S 3$, разд. 1, вида (без петель), $d \rho(\zeta)$ - борелевская вероятностная мера на ней, $m(z)$ - соответствующая функция Вейля

$$
m(z)=\int_{S} \frac{d \rho(\zeta)}{\zeta-z}, \quad z \in \mathbb{C} \backslash S .
$$

Утверждается, что $m(z)$ однозначно определяет меру $d \rho(\zeta)$. 
В самом деле, по аналогии с доказательством теоремы 5 преобразуем (46) следующим образом. При $|z| \geqslant R$, где $R>0$ достаточно большое,

$$
\begin{gathered}
m(z)=-\frac{1}{z} \int_{S} \frac{d \rho(\zeta)}{1-\zeta z^{-1}}=-\frac{1}{z} \int_{S}\left(\sum_{n=0}^{\infty}\left(\frac{\zeta}{z}\right)^{n}\right) d \rho(\zeta)=-\frac{1}{z} \sum_{n=0}^{\infty} \frac{s_{n}}{z^{n}}, \\
s_{n}=\int_{S} \zeta^{n} d \rho(\zeta), \quad n \in \mathbb{N}_{0} .
\end{gathered}
$$

Из этого разложения следует, что функция $m(z), z \in \mathbb{C} \backslash S$, однозначно определяет моменты $s_{n}$ и тем самым для каждого полинома $P(\zeta)$ от $\zeta$ с комплексными коэффициентами - интеграл $\int_{S} P(\zeta) d \rho(\zeta)$. Но такие полиномы плотны в пространстве всех непрерывных функций $f(\zeta)$ на $S$ в равномерной метрике (см., например, [43, гл. 5, §4]). Отсюда следует, что любой такой интеграл $\int_{S} f(\zeta) d \rho(\zeta)$, а значит, и мера $d \rho(\zeta)$ по $m(z)$ определяются однозначно.

Замечание 2. Аналогичный приведенному в замечании 1 факт в случае, когда $S=\mathbb{T}$ - единичная окружность, доказывается иначе.

А именно, введем функцию Каратеодори

$$
F(z)=\int_{\mathbb{T}} \frac{\zeta+z}{\zeta-z} d \rho(\zeta), \quad z \in \mathbb{C} \backslash \mathbb{T} .
$$

Она определяет меру $d \rho(\zeta)$ однозначно; см., например, [36, гл. 1, разд. 1.3] и [41]. Но $F(z)=2 m(z)+1, z \in \mathbb{C} \backslash \mathbb{T}$.

Для деформации окружности нужно воспользоваться ее конформным отображением на $\mathbb{T}$.

2. Перейдем к рассмотрению уравнения Лакса (17) в случае, когда $J(t)$ имеет указанный выше (в разд. 1) вид, и выясним возможности его интегрирования.

Лемма 3. Для функиии $M(z ; t)$ вида (43) и оператора $A(t)$, порожсденного матрицей (19), справедливо равенство

$$
\begin{aligned}
\dot{M}(z ; t)=( & \left.R_{z}(t) A(t) \delta_{0}, R_{z}(t) \delta_{0}\right)_{\mathbf{l}_{2}}+\left(R_{z}(t) \delta_{0}, R_{z}(t) A(t) \delta_{0}\right)_{\mathbf{l}_{2}} \\
& -\left(\left(A(t)+A^{+}(t)\right) R_{z}(t) \delta_{0}, R_{z}(t) \delta_{0}\right)_{\mathbf{l}_{2}}, \quad z \in \mathbb{C} \backslash S, t \in[0, T] .
\end{aligned}
$$

Доказательство. Используя (25), получим

$$
\begin{aligned}
\dot{M}(z ; t)= & \left(R_{z}(t) \delta_{0}, R_{z}(t) \delta_{0}\right)_{\mathbf{l}_{2}}=\left(\dot{R}_{z}(t) \delta_{0}, R_{z}(t) \delta_{0}\right)_{\mathbf{l}_{2}}+\left(R_{z}(t) \delta_{0}, \dot{R}_{z}(t) \delta_{0}\right)_{\mathbf{l}_{2}} \\
= & \left(R_{z}(t) A(t) \delta_{0}-A(t) R_{z}(t) \delta_{0}, R_{z}(t) \delta_{0}\right)_{\mathbf{l}_{2}} \\
& \quad+\left(R_{z}(t) \delta_{0}, R_{z}(t) A(t) \delta_{0}-A(t) R_{z}(t) \delta_{0}\right)_{\mathbf{l}_{2}} \\
= & \left(R_{z}(t) A(t) \delta_{0}, R_{z}(t) \delta_{0}\right)_{\mathbf{l}_{2}}+\left(R_{z}(t) \delta_{0}, R_{z}(t) A(t) \delta_{0}\right)_{\mathbf{l}_{2}} \\
& \quad-\left(\left(A(t)+A^{+}(t)\right) R_{z}(t) \delta_{0}, R_{z}(t) \delta_{0}\right)_{\mathbf{l}_{2}} .
\end{aligned}
$$

Для задания оператора $A(t)$ напомним, что, согласно $\S 1$, разд. 2 , в пространстве $\mathbf{l}_{2}$ ортонормированным базисом будет последовательность векторов

$$
\delta_{0}=\delta_{0 ; 0} ; \delta_{1 ; 0}, \delta_{1 ; 1} ; \delta_{2 ; 0}, \delta_{2 ; 1}, \delta_{2 ; 2} ; \ldots ; \delta_{n ; 0}, \delta_{n ; 1}, \ldots, \delta_{n ; n} ; \ldots, \quad n \in \mathbb{N}_{0}
$$


В этом базисе для любых $f, g \in \mathbf{l}_{2}$ справедливо разложение

$$
(f, g)_{\mathbf{l}_{2}}=\sum_{n=0}^{\infty} \sum_{\alpha=0}^{n}\left(f, \delta_{n ; \alpha}\right)_{\mathbf{l}_{2}} \overline{\left(g, \delta_{n ; \alpha}\right) \mathbf{l}_{2}} .
$$

Лемма 4. Предположим, что у рассматриваемой матрииы $J(t)$ элемент $c_{0}(t)$ равен нулю, а $A(t)$ такова, что

$$
\gamma_{n}(t)=-\alpha_{n}^{+}(t), \quad n \in \mathbb{N}_{0} ; \quad \alpha_{0}(t)=0 ; \quad \beta_{n}(t)=\beta(t) \mathbf{1}_{n}, \quad n \in \mathbb{N}, t \in[0, T] .
$$

Здесь $\beta(t)$ - некоторая заданная функция из пространства $C([0, T]), a \mathbf{1}_{n}$ единичный оператор в пространстве $\mathscr{H}_{n}$.

Тогда функиия $M(z ; t)($ см. (43)) удовлетворяет уравнению

$$
\dot{M}(z ; t)=2 \operatorname{Re}\left(\beta_{0}(t)-\beta(t)\right)(M(z ; t)-m(z ; t) \overline{m(z ; t)}),
$$

где $z \in \mathbb{C} \backslash S, t \in[0, T], m(z ; t)$ - функиия Вейля $(22)$ оператора $\boldsymbol{J}(t)$.

Доказательство. Согласно (50), матрица $A(t)+A^{+}(t)$ является блочной диагональной матрицей с элементами

$$
2 \operatorname{Re} \beta_{0}(t), 2(\operatorname{Re} \beta(t)) \mathbf{1}_{1}, 2(\operatorname{Re} \beta(t)) \mathbf{1}_{2}, \ldots
$$

на диагонали, причем $A(t) \delta_{0}=\beta_{0}(t) \delta_{0}, A^{+}(t) \delta_{0}=\overline{\beta_{0}(t)} \delta_{0}$. Раскладывая $M(z ; t)=$ $\left(R_{z}(t) \delta_{0}, R_{z}(t) \delta_{0}\right)_{\mathbf{l}_{2}}$, согласно равенству (49), по базису (48) и пользуясь определением функции Вейля $m(z ; t)$, на основании $(47)$ получаем

$$
\begin{aligned}
\dot{M}(z ; t)= & 2\left(\beta_{0}(\mathrm{t})\right)\left(M(z ; t)-\left(R_{z} \delta_{0}, \delta_{0}\right)_{\mathbf{l}_{2}} \overline{\left(R_{z} \delta_{0}, \delta_{0}\right)_{\mathbf{l}_{2}}}\right) \\
& -2(\operatorname{Re} \beta(t)) \sum_{n=1}^{\infty} \sum_{\alpha=0}^{n}\left(R_{z}(t) \delta_{0}, \delta_{n ; \alpha}\right)_{\mathbf{l}_{2}} \overline{\left(R_{z}(t) \delta_{0}, \delta_{n ; \alpha}\right)_{\mathbf{l}_{2}}} \\
= & 2\left(\operatorname{Re}\left(\beta_{0}(t)-\beta(t)\right)\right)(M(z ; t)-m(z ; t) \overline{m(z ; t)}) .
\end{aligned}
$$

Из доказательства теоремы 3 следует, что вывод уравнения для функции Вейля сохраняется и в рассматриваемом сейчас случае неодномерного спектра оператора $\boldsymbol{J}(t)$. Поэтому функция $m(z ; t)$ из $(51)$ удовлетворяет прежнему уравнению (30): условия леммы 4 обеспечивают выполнение условий (29) теоремы 3. Поэтому и сейчас справедлив факт, указанный в начале §3, разд. 3. Это приводит к следующему результату.

Теорема 6. Рассмотрим систему нелинейных уравнений

$$
\begin{array}{lll}
\dot{a}_{n}=b_{n+1} c_{n}^{+}-c_{n}^{+} b_{n}, \quad n \in \mathbb{N}, \quad \dot{a}_{0}=\left(\beta_{0}-\beta\right) a_{0}, & a_{n}: \mathscr{H}_{n} \rightarrow \mathscr{H}_{n+1}, \\
\dot{b}_{n}=c_{n} a_{n}-a_{n-1} c_{n-1}+c_{n} c_{n}^{+}-c_{n-1}^{+} c_{n-1}, \quad n \in \mathbb{N}_{0}, & b_{n}: \mathscr{H}_{n} \rightarrow \mathscr{H}_{n}, \\
\dot{c}_{n}=c_{n} b_{n+1}-b_{n} c_{n}, \quad a_{n+1} c_{n}^{+}=c_{n+1}^{+} a_{n}, \quad n \in \mathbb{N}, & c_{n}: \mathscr{H}_{n+1} \rightarrow \mathscr{H}_{n} .
\end{array}
$$

Здесъ $t \in[0, T], T \leqslant \infty, a_{-1}=c_{-1}=0, \beta_{0}, \beta \in C([0, T])$ заданы.

Пусть существует решение $\left(a_{n}(t)\right)_{n=0}^{\infty},\left(b_{n}(t)\right)_{n=0}^{\infty},\left(c_{n}(t)\right)_{n=0}^{\infty}$ задачи Коши для этой системы, такое, что матрица (5) порождает нормальный ограниченный оператор $\boldsymbol{J}(t)$ в пространстве $\mathbf{l}_{2}$, для которого $a_{0}=c_{0}=0$. Тогда это решение восстанавливается при помощи формулы (16), в которой спектральная мера $d \rho(\zeta ; t)$ находится по началъной спектралъной мере $d \rho(\zeta ; 0)$ следующей процедурой: 1) по $d \rho(\zeta ; 0)$ находим бункцию Вейля $m(z ; 0), z \in \mathbb{C} \backslash S$, 
и затем решаем задачу Коши (30) с начальными данными $m(z ; 0)$; 2) решаем задачу Коши для уравнения (51) с начальными даннъми $M(z ; 0)$, которые находятся по $d \rho(\zeta ; 0)$ согласно $(44) ; 3)$ найденная функиия $M(z ; t), z \in \mathbb{C} \backslash S$, $t \in[0, T]$, однозначно определяет меру $d \rho(\zeta ; t)$ согласно теореме 5.

Доказательство. Рассмотрим матрицу (19), в которой

$$
\begin{gathered}
\alpha_{n}=c_{n}^{+}, \quad \gamma_{n}=-\alpha_{n}^{+}=-c_{n}, \quad n \in \mathbb{N}_{0}, \quad c_{0}=0, \\
\beta_{n}=\beta \mathbf{1}_{n}, \quad n \in \mathbb{N}, \quad \beta, \beta_{0} \in C([0, T]) .
\end{gathered}
$$

Соотношения (53) влекут за собой выполнение условий леммы 4, и поэтому функция $M(z ; t)$ удовлетворяет уравнению (51). Уравнение $(30)$ для $m(z ; t)$ также будет выполняться, так как из условий доказываемой теоремы вытекают требования (29) (с $\left.c_{0}=0\right)$. Таким образом, осталось убедиться, что равенства (53) и $a_{0}=0$ приводят к тому, что система (21) преобразуется в систему (52). Это делается элементарно.

Отметим также, что и в рассматриваемом случае двумерного спектра можно доказать аналог теоремы 4 - построить решение задачи Коши для системы (52) по начальным данным, которые задаются посредством меры $d \rho(\zeta ; 0)$. Доказательство подобного утверждения достаточно громоздко и будет приведено в другом месте.

В заключение авторы выражают искреннюю благодарность Л. Б. Голинскому за стимулирующие беседы и И. М. Кричеверу за полезные замечания.

\section{ЛитерАТУРА}

[1] Б. М. Левитан, Обратнъе задачи Штурма-Лиувилля, Наука, М., 1984.

[2] M. Kac, P. van Moerbeke, On an explicitly soluble system of nonlinear differential equations related to certain Toda lattices, Adv. Math., 16:2 (1975), 160-169.

[3] J. Moser, Three integrable Hamiltonian systems connected with isospectral deformations, Adv. Math., 16:2 (1975), 197-220.

[4] Yu. M. Berezansky, The integration of semi-infinite Toda chain by means of inverse spectral problem, Preprint 84.79 of Inst. of Math., Acad. Sci. Ukr., Kiev, 1984.

[5] Ю. М. Березанский, Интегрирование нелинейных разностных уравнений методом обратной спектралъной задачи, Докл. АН СССР, 281:1 (1985), 16-19.

[6] Yu. M. Berezanski, The integration of semi-infinite Toda chain by means of inverse spectral problem, Rep. Math. Phys., 24:1 (1986), 21-47.

[7] Yu. M. Berezansky, M. E. Dudkin, The direct and inverse spectral problems for the block Jacobi type unitary matrices, Methods Funct. Anal. Topology, 11:4 (2005), 327345.

[8] Yu. M. Berezansky, M. E. Dudkin, The complex moment problem and direct and inverse spectral problems for the block Jacobi type bounded normal matrices, Methods Funct. Anal. Topology, 12:1 (2006), 1-31.

[9] М. Г. Крейн, Бесконечные J-матричъ и матричная проблема моментов, Докл. AH CCCP, 69:2 (1949), 125-128.

[10] Ю. М. Березанский, Разложение по собственным функииям уравнений в частных разностях второго порядка, Труды Моск. матем. об-ва, 5 (1956), 203-268.

[11] В. Г. Тарнопольський, Про самоспряженість різнищевих операторів з операторними коебіцієнтами, ДАН УРСР, 11 (1959), 1189-1192.

[12] Ю. М. Березанский, Разложение по собственным функииям самосопряженных операторов, Наукова думка, Киев, 1965. 
[13] Ю. М. Березанский, М. И. Гехтман, Обратная задача спектралъного анализа и неабелевы цепочки нелинейных уравнений, Укр. матем. ж., 42:6 (1990), 730-747.

[14] М. И. Гехтман, Интегрирование неабелевых цепочек типа Тоды, Функц. анализ и его прил., 24:3 (1990), 72-73.

[15] L. Faybusovich, M. Gekhtman, Elementary Toda orbits and integrable lattices, J. Math. Phys., 41:5 (2000), 2905-2921.

[16] Ю. М. Березанский, Прямая и обратная спектралъные задачи для якобиева поля, Алгебра и анализ, 9:6 (1997), 38-61.

[17] Ю. Л. Кишакевич, Спектральная функиия типа Марченко разностного оператора четного порядка, Матем. заметки, 11:4 (1972), 437-446.

[18] Ю. Л. Кишакевич, Спектралъная функиия типа Марченко бесконечной системъ разностных уравнений, Теория функций, функц. анализ и их приложения, 16 (1972), 59-68.

[19] И. М. Кричевер, Периодическая неабелева цепочка Тода и ее двумерное обобщение, Приложение (с. 72-80) к статъе: Б. А. Дубровин, Тэта-функции и нелинейные уравнения, УМH, 36:2 (1981), 11-80.

[20] M. Kac, P. van Moerbeke, On some periodic Toda lattices, Proc. Nat. Acad. Sci. USA, 72:4 (1975), 1627-1629.

[21] С. В. Манаков, О полной интегрируемости и стохастизации в дискретных динамических системах, Журн. эксп. теорет. физ., 67:2 (1974), 543-555.

[22] H. Flaschka, On the Toda lattice. I, Phys. Rev., B, 9 (1974), 1924-1925.

[23] H. Flaschka, On the Toda lattice. II, Progr. Theoret. Phys., 51:3 (1974), 703-716.

[24] Н. В. Жернаков, Прямая и обратная задачи для периодической якобиевой матрицы, Укр. матем. ж., 38:6 (1986), 785-788.

[25] Н. В. Жернаков, Интегрирование цепочек Тодь в классе операторов ГилъбертаШмидта, Укр. матем. ж., 39:5 (1987), 645-648.

[26] Н. В. Жернаков, Спектральная теория некоторых классов якобиевых матрии, и ее применения к интегрированию цепочки Тодь, Дисс. к.ф.-м.н., Институт математики АН УССР, Киев, 1987.

[27] M. Bruschi, S. V. Manakov, O. Ragnisco, D. Levi, The nonabelian Toda lattice - discrete analogue of the matrix Schrödinger spectral problem, J. Math. Phys., 21 (1980), 2749-2753.

[28] M. Bruschi, O. Ragnisco, D. Levi, Evolution equations associated with the discrete analog of the matrix Schrödinger spectral problem solvable by the inverse spectral transform, J. Math. Phys., 22 (1981), 2463-2471.

[29] А. Ю. Далецкий, Г. Б. Подколзин, Групповой подход к интегрированию бесконечной иепочки Тодь, Укр. матем. ж., 40:4 (1988), 518-521.

[30] Yu. M. Berezansky, Integration of nonlinear nonisospectral difference-differential equations by means of the inverse spectral problem, in: Nonlinear Physics, Theory and Experiment (Proc. of the First Workshop, Gallipoli (Lecce), Italy, June 29-July 7, 1995), World Sci. Publ., River Edge, NJ, 1996, 11-20.

[31] G. Teschl, Jacobi Operators and Completely Integrable Nonlinear Lattices, in: Mathematical Surveys and Monographs, vol. 72, Amer. Math. Soc., Providence, RI, 2000.

[32] Ю. М. Березанский, М. И. Гехтман, М. Е. Шмойш, Интегрирование методом обратной спектральной задачи некоторых чепочек нелинейных разностных уравнений, Укр. матем. ж., 38:1 (1986), 84-89.

[33] Yu. Berezansky, M. Shmoish, Nonisospectral flows on semi-infinite Jacobi matrices, Nonlinear Math. Phys., 1:2 (1994), 116-146.

[34] О. А. Мохонько, Деякі розв'язні класи нелінійних неізоспектральних різницевих рівнянъ, Укр. матем. ж., 57:3 (2005), 356-365. 
[35] M. Szczepanik, Integration of semi-infinite Toda chain in some class of unbounded solutions, Rep. Math. Phys., 39:2 (1997), 263-273.

[36] B. Simon, Orthogonal Polynomials on the Unit Circle. Part 1: Classical Theory, Part 2: Spectral Theory, Amer. Math. Soc. Colloquium Publications, Amer. Math. Soc., Providence, RI, 2005.

[37] B. Simon, OPUC on one foot, Preprint, Mathematics 253-37, California Institute of Technology, Pasadena, CA 91125, 2005.

[38] M. J. Cantero, L. Moral, L. Velázquez, Five-diagonal matrices and zeros of orthogonal polynomials on the unite circle, Linear Algebra Appl., 362 (2003), 29-56.

[39] Л. Б. Голинский, Потоки Шура и ортогоналъные полиномы на единичной окружности, Матем. сб., 197:8 (2006), 41-62.

[40] И. И. Привалов, Граничные свойства аналитических функиий, Гостехиздат, М.Л., 1950.

[41] W. Rudin, Real and Complex Analysis, McGraw-Hill, New York, 1987.

[42] Yu. M. Berezansky, M. E. Dudkin, On the complex moment problem, Math. Nachr., 280:1-2 (2007), 60-73.

[43] А. И. Маркушевич, Теория аналитических функиий, Гостехиздат, М.-Л., 1950.

Институт математики НАН Украины e-mail: berezan@mathber.carrier.kiev.ua

Поступило в редакцию Киевский национальный университет имени Тараса Шевченко 29 мая 2007 г. e-mail: AlexeyMohonko@univ.kiev.ua 